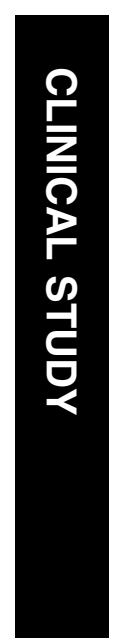

Department of

Ophthalmology,

Hadassah-Hebrew

University

Medical Center,

Jerusalem, Israel

Correspondence:

EZ Blumenthal,

Department of

Ophthalmology,

Hadassah University

Hospital,

PO Box 12000 ,

Jerusalem 91120,

Israel

Tel: + 9722677 6580;

Fax: +972 26778852

E-mail: eytan@blumenthal. org.il

Received: 23 August 2006 Accepted in revised form: 4 May 2007

Published online: 13 July 2007

\section{Clinical \\ characteristics of eyes demonstrating atypical patterns in scanning laser polarimetry}

A Orlev, A Horani, Y Rapson, MJ Cohen and EZ Blumenthal

\begin{abstract}
Purpose To characterize which clinical features are associated with the occurrence of atypical birefringence patterns (ABP) occasionally seen with scanning laser polarimetry (SLP).

Methods Sixty-one subjects, including glaucoma patients, glaucoma suspects, and normal subjects, underwent a full clinical examination, standard visual field (VF) test, and a GDx-VCC SLP examination. One eye was selected from each patient. The magnitude of ABP was determined in two independent ways: using a support vector machine analysis (typical scan score (TSS)) and by a masked experienced observer. We assessed whether the magnitude of ABP was correlated with age, gender, the refractive state of the eye, corneal polarization axis and magnitude, GDx global parameters (TSNIT and NFI), and the VF status, as evident from glaucoma hemifield test (GHT), mean deviation (MD), and the pattern standard deviation (PSD).

Results Of the 61 study eyes, 27 (44\%)

showed an ABP, based on a TSS cutoff $(<82.5)$. A very high correlation was found between the TSS score and the masked experienced observer score $\left(r^{2}=0.80 ; P<0.001\right)$. The following clinical parameters were found, on bivariate analysis, to be significantly correlated with the presence of an ABP: age $\left(r^{2}=0.086 ; P=0.02\right)$; corneal polarization magnitude $\left(r^{2}=0.069 ; P=0.04\right)$; TSNIT ( $\left.r^{2}=0.16 ; P<0.001\right)$.

Conclusion The presence and magnitude of ABP did not seem to be closely correlated with most clinical parameters. A low, but statistically significant, correlation was found for age and corneal polarization magnitude ( $r^{2}=0.086$ and 0.069 , respectively). A low-

medium correlation was found for TSNIT $\left(r^{2}=0.16\right)$; however, we speculate that this might represent a confounding effect, rather than an underlying association. We conclude that none of the clinical parameters investigated in this study appears to be strongly correlated with the presence of an ABP on SLP scans performed using the commercially available GDx-VCC.

Eye (2008) 22, 1378-1383; doi:10.1038/sj.eye.6702897; published online 13 July 2007

Keywords: glaucoma imaging; glaucoma diagnosis; retinal nerve fiber layer; scanning laser polarimetry; atypical birefringence pattern; GDx-VCC

Introduction

Scanning laser polarimetry (SLP) is an imaging modality utilized for diagnosing glaucoma ${ }^{1,2}$ and for follow-up of established glaucoma. SLP combines a confocal scanning laser ophthalmoscope with an integrated polarimeter designed to measure the peripapillary retinal nerve fibre layer (RNFL) thickness. Polarized light undergoes a phase shift, also called retardation, as it passes through polarizing media, such as the cornea, the crystalline lens, and the RNFL. This phase shift was previously shown to correlate with the thickness of the RNFL. ${ }^{3}$

The introduction of custom corneal compensation, initially in prototype mode and later as the GDx-VCC commercial model, ${ }^{4,5}$ addressed the need for individualized corneal compensation, based on the corneal birefringence axis and magnitude $e^{6-8}$ of each
\end{abstract}


scanned eye. The GDx-VCC incorporates a two-step approach to compensate for the retardation component derived from the polarizing properties of the anterior structures of the eye. This is achieved by first scanning the foveal area, attributing any non-uniform light shift to anterior segment (cornea and lens) polarization. ${ }^{4}$ In the second step, a compensated scan of the peripapillary retina is acquired. SLP was shown to be a highly reproducible imaging technique of the RNFL. ${ }^{9-11}$

After experience with the GDx-VCC accumulated, it became apparent that an additional SLP anomaly, which is likely unrelated to corneal compensation, appears in a small proportion of scanned individuals. ${ }^{12}$ This SLP anomaly, or artefact, became known as an atypical birefringence pattern (ABP), and consequently has been addressed by the manufacturer via a typical scan score (TSS) score (on a scale of 0-100), such that normal patterns score high, while ABP scans score low. Once familiar with this unusual pattern, the ABP can be relatively easy to recognize in a subset of scans, as patchy peripapillary retardation, usually seen in a circumferential, combined with a radial, configuration. The ABP differs considerably from the normal, healthy, pattern corresponding to the arcuate nerve fibre bundles (Figure 1).

It is, at present, unclear what the underlying cause of $\mathrm{ABP}$ is. High reproducibility of this artefact suggests that it is likely a characteristic present within the scanned eye, most likely at the posterior pole of the eye, rather than a machine malfunction or faulty eye-machine interaction. Bagga et al ${ }^{12}$ discuss several hypotheses for the underlying cause of ABP, but conclude that 'the pathophysiology of ABP images is unknown'.
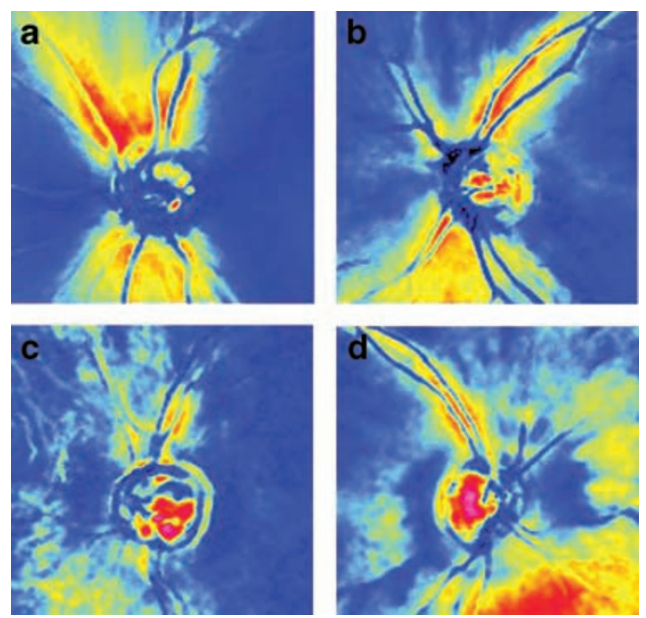

Figure 1 Examples of normal and ABP scans (GDx-VCC): (a) no atypia (TSS $=100)$; (b) mild atypia (TSS $=79$ ); (c) moderate atypia $($ TSS $=67)$; $(d)$ severe atypia $($ TSS $=5$ ).
In this study, we set to identify which clinical characteristics might correlate with the presence of ABP and TSS, and to identify any correlation with glaucoma severity, as evident by three global VF parameters.

\section{Materials and methods}

\section{Subjects}

Sixty-one glaucoma patients, glaucoma suspects, and normal subjects, all Caucasians, were recruited from a large tertiary glaucoma clinic, as well as hospital personnel and normal volunteers. Recruited subjects were classified in this study based on the visual field (VF) results, to avoid classification based on structural (disc and/or RNFL) criteria. Based on the VF Glaucoma Hemifield Test (GHT), 25 subjects were within normal limits (WNL), nine were on the borderline, and 27 were outside normal limits (ONL).

Inclusion criteria included visual acuity of $20 / 40$ or better, and age over 18. Exclusion criteria included any prior intraocular or refractive surgery, a known neuroophthalmological condition, diabetic retinopathy, or poor reliability of the VF (defined as fixation losses, falsepositive or false-negative errors $>33 \%$ ).

One eye was enrolled for each subject. In those subjects in whom both eyes qualified, the eye with the lower TSS was recruited, to increase the sensitivity of our analysis.

Each patient underwent an eye examination, including a slit-lamp evaluation of the anterior segment, gonioscopy of the angle, Goldmann applanation tonometry, and a dilated stereoscopic examination of the optic disc and fundus, to exclude non-glaucomatous reasons for VF loss or posterior pole abnormalities. Each underwent a standard, white on white, 24-2 SITA standard VF examination, as well as a GDx-VCC scan, within 6 months of each other. The mean time interval between the VF and the GDx-VCC test was $36 \pm 56$ days, such that $34 \%$ of the subjects had two tests performed on the same day and $69 \%$ within a month.

\section{GDx-VCC imaging}

GDx-VCC (Carl Zeiss-Meditec, Dublin, CA, USA, formerly Laser Diagnostic Technologies, San Diego, CA, USA) was performed by a single experienced technician, using the standard $3.2 \mathrm{~mm}$ circular scan protocol, centred on the optic disc. Since we noted that highly ABP eyes often tended to receive a low-quality scan score, we refrained from excluding eyes merely on the basis of a low-quality score. Instead, we relied on qualitative evaluation of scan quality, noting illumination, motion artefacts, blinking artefacts, unfocused images, and poorly centred images. 


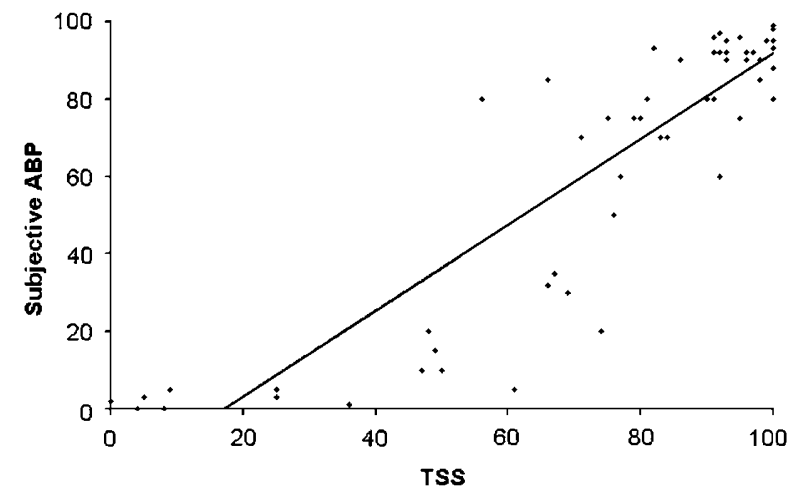

Figure 2 Machine-determined TSS compared with the subjective ABP score, obtained by a masked observer $\left(r^{2}=0.8\right.$; $P<0.001)$

\section{Scoring the magnitude of $A B P$}

\section{TSS}

Machine-determined score, referred to by the manufacturer as 'typical scan score', is based on a support vector machine analysis. This analysis is a builtin feature of the GDx software, and is accessed by exporting a raw data spreadsheet file.

\section{Subjective $A B P$ score}

This is a subjective score, as determined by a masked experienced observer. A single experienced observer, masked to all clinical information, was shown isolated colour thickness maps, and scored each map on a scale of $0-100$, with the aid of a standardized set of nine TSS scored examples, appearing as Figure 2, in an article by Bagga et al. ${ }^{12}$ By comparing each masked thickness map to the examples appearing in this figure, the ABP component in each thickness map was subjectively scored.

\section{Statistical analysis}

Correlation between continuous variables and TSS values was calculated using Spearman's $\rho$ coefficient. TSS values between males and females were assessed using Student's $t$-test. TSS values between the different GHT groups were assessed with analysis of variance (ANOVA) using the $F$ statistics. $P<0.05$ were deemed statistically significant. Stepwise conditional backward logistic regression was employed to predict which variables correlated with ABP.

\section{Results}

Patient characteristics are summarized in Table 1.

\section{Machine-determined TSS vs ABP score}

When comparing the machine-determined TSS to the subjective ABP score, the two scoring systems were
Table 1 Patient's clinical characteristics

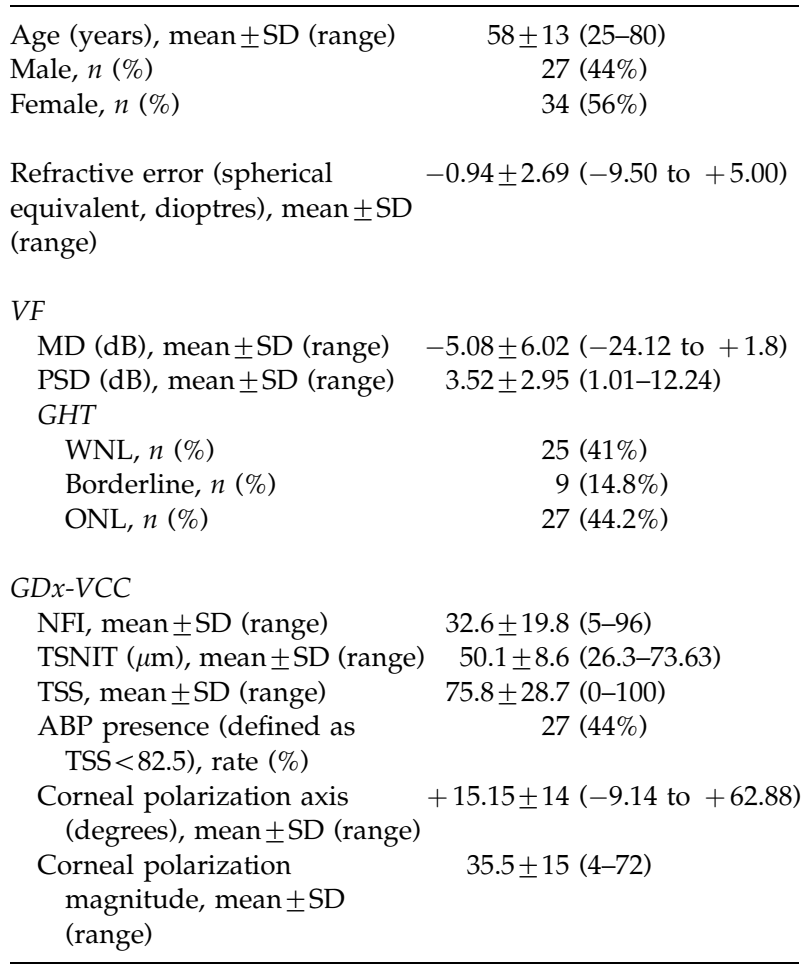

$\mathrm{ABP}$, atypical birefringence pattern; GHT, glaucoma hemifield test; MD, mean deviation; NFI, nerve fibre indicator; ONL, outside normal limits; PSD, pattern standard deviation; TSNIT, temporal-superior-nasal-inferior-temporal; TSS, typical scan score; WNL, within normal limits.

found to be highly correlated $\left(r^{2}=0.8, P<0.001\right)$. Figure 2 is a scatterplot of this data, along with a regression line.

\section{Correlating the magnitude of ABP to clinical characteristics}

Table 2 contains a bivariate analysis of the TSS score with various clinical characteristics. Of the 10 clinical parameters studied, only age and temporal-superiornasal-inferior-temporal (TSNIT) were found, on bivariate analysis, to be significantly correlated with TSS: age $\left(r^{2}=0.066 ; P=0.04\right)$; TSNIT $\left(r^{2}=0.16 ; P<0.001\right)$. An almost significant correlation was found between TSS and corneal polarization magnitude $\left(r^{2}=0.062\right.$; $P=0.053)$. Figure 3 demonstrates the association between age, refractive error, TSNIT, and corneal polarization magnitude with TSS. TSS score was previously defined as ABP with values lower than 82.5 , and normal birefringence pattern with TSS above 82.5 according to Bagga et al. ${ }^{12}$ Using this dichotomous criterion, logistic regression analysis of our data found that nerve fibre indicator (NFI) and TSNIT were significantly associated with $\mathrm{ABP}(\mathrm{OR}=0.9,95 \% \mathrm{CI}$ : 0.85-0.98, $P=0.009$; $\mathrm{OR}=0.7,95 \% \mathrm{CI}: 0.6-0.88, P=0.001$, respectively). 


\section{Discussion}

The GDx-VCC is a scanning laser polarimeter that allows measurement of the RNFL thickness at the posterior pole. However, the recognition of anomalous patterns, called ABPs, in a subset of healthy as well as glaucomatous

Table 2 Bivariate analysis, correlating TSS with clinical, functional, and structural characteristics

\begin{tabular}{lcc}
\hline & \multicolumn{2}{c}{ Correlation with TSS } \\
\cline { 2 - 3 } & \multicolumn{1}{c}{$\mathrm{r}^{2}$} & P-value \\
\hline Clinical characteristics & & \\
Age & 0.066 & 0.04 \\
Male : female & - & $0.4^{\mathrm{a}}$ \\
Refractive error & 0.0002 & 0.9 \\
Functional characteristics (VF) & & \\
MD & 0.015 & 0.33 \\
PSD & 0.03 & 0.19 \\
GHT & - & $0.384^{\mathrm{b}}$ \\
Structural characteristics (GDx-VCC) & & \\
NFI & 0.025 & 0.216 \\
TSNIT & 0.16 & $<0.001$ \\
Corneal polarization axis & 0.006 & 0.53 \\
Corneal polarization magnitude & 0.062 & 0.053 \\
\hline
\end{tabular}

GHT, glaucoma hemifield test; MD, mean deviation; NFI, nerve fibre indicator; PSD, pattern standard deviation; TSNIT, temporal-superiornasal-inferior-temporal; TSS, typical scan score; VF, visual field.

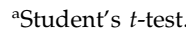

${ }^{\mathrm{b}}$ ANOVA (F-test). eyes, deserves particular attention to allow better interpretation of GDx-VCC images and the RNFL data derived from the use of this device. It appears that a subset of GDx-VCC scans is entirely uninterpretable, while others are partially interpretable. ${ }^{12}$ The underlying cause of $\mathrm{ABP}$ is still unknown. It was postulated that ABP might perhaps result from a low signal-to-noise ratio stemming from loss of pigmented epithelium reflectivity in these eyes. Bagga et $a l^{12}$ demonstrated that ABPs appeared more frequently with older age and in Caucasian eyes with glaucoma.

DaPozzo et al, ${ }^{13}$ studying eyes showing APBs, found that NFI and other GDx-VCC parameters that are independent of absolute RNFL thickness, such as modulation, ratios, and standard deviation, show better diagnostic accuracy when applied to those eyes demonstrating ABPs. These two prior studies characterized the presence or lack of an ABP, as well as the presence or lack of glaucoma, in a binary (yes-no) classification. ${ }^{12,13}$ In contrast, we chose to present the magnitude of ABP, as well as the extent of glaucomatous damage, on a continuous scale, to better capture any correlations present in the data. ${ }^{14}$

In this study, we evaluated which clinical characteristics correlated with ABP on SLP imaging. While age and corneal polarization magnitude associations with TSS are of clinical interest, the association of TSNIT and TSS was expected. We speculated that since eyes showing ABP demonstrate a blotchy pattern of peripapillary RNFL thickening, this
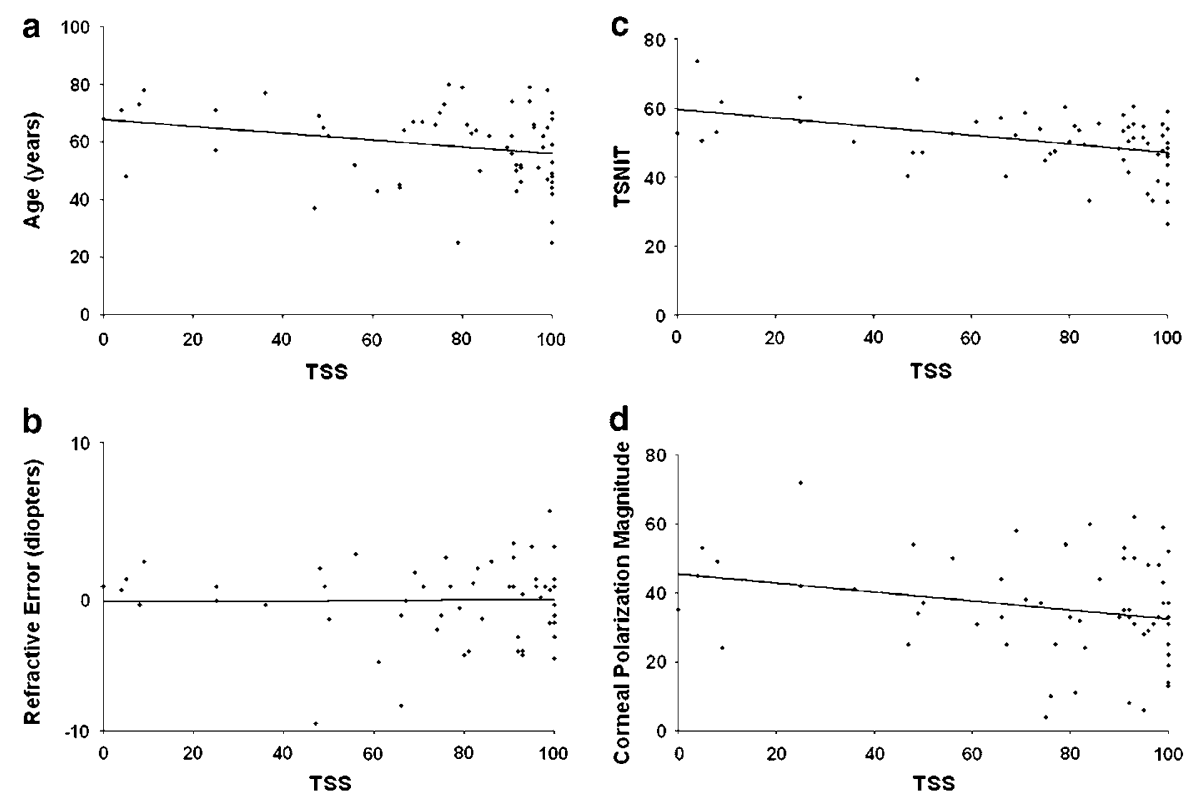

Figure 3 TSS compared with (a) age $\left(r^{2}=0.066 ; P=0.04\right)$, (b) refractive error $\left(r^{2}=0.0002 ; P=0.9\right)$, (c) TSNIT $\left(r^{2}=0.16 ; P<0.001\right)$, and (d) corneal polarization magnitude $\left(r^{2}=0.062 ; P=0.053\right)$. 

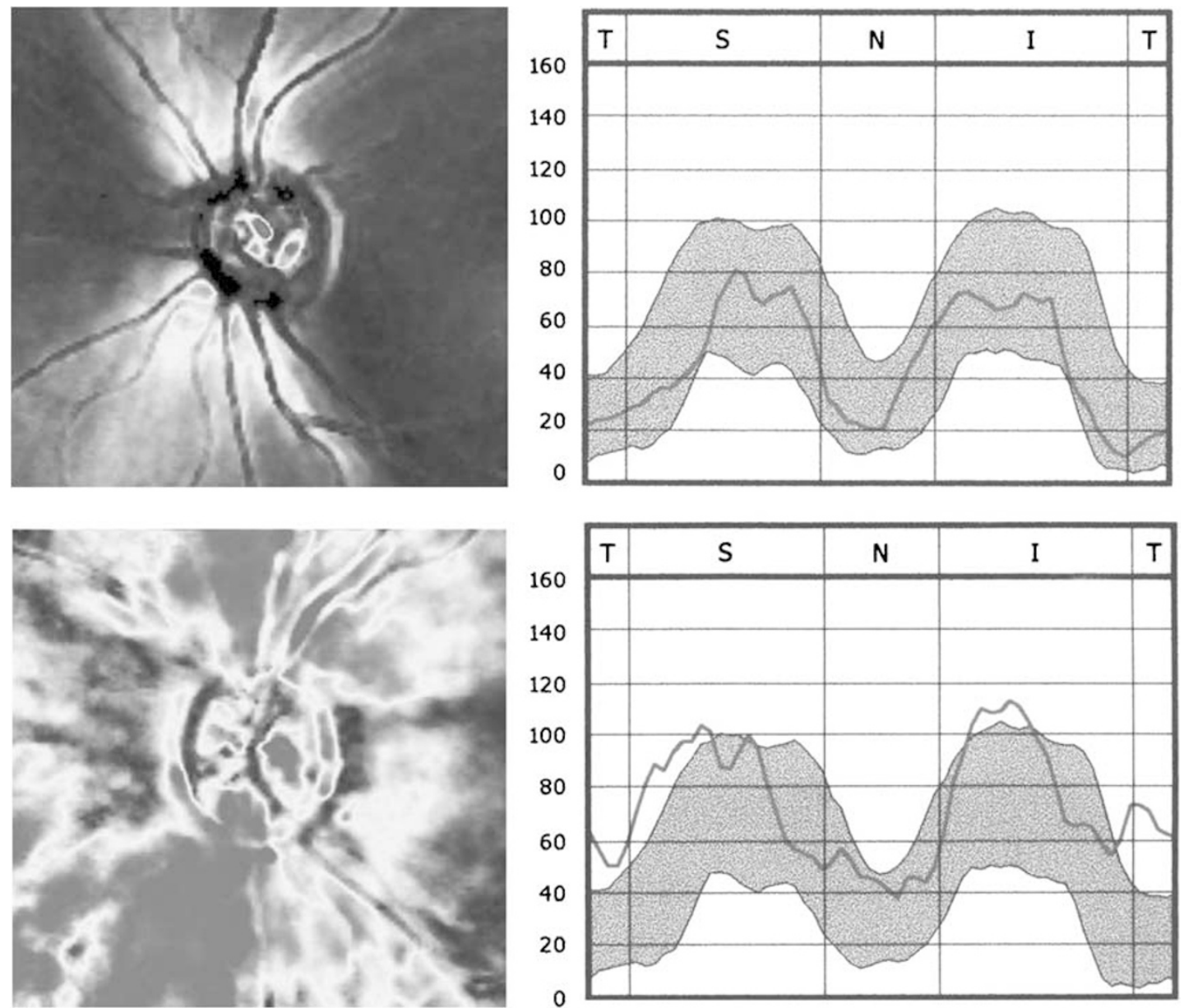

Figure 4 An example of two normal eyes (top) without atypia (TSS =98) and (bottom) with atypia (TSS=4). Note that the TSNIT graph of the eye with atypia is displaced up owing to the atypia component, hence translating into higher RNFL values throughout the TSNIT graph.

would likely translate into an artefactiously increased TSNIT value in those eyes (Figure 4).

While we did not find an association between refractive error and TSS in our study sample, our study cannot rule out that large refractive errors might correlate with ABP.

In conclusion, more research on the source of atypia is needed, to try and unravel the causative factors underlying this artefact and to characterize those eyes that are most likely to demonstrate this abnormal pattern of birefringence.

\section{Acknowledgements}

EZB has received research support from Laser Diagnostic Technologies Incorporated.

\section{References}

1 Zangwill LM, Bowd C. Retinal nerve fiber layer analysis in the diagnosis of glaucoma. Curr Opin Ophthalmol 2006; 17: $120-131$.
2 Reus NJ, Lemij HG. Relationships between standard automated perimetry, HRT confocal scanning laser ophthalmoscopy, and GDx VCC scanning laser polarimetry. Invest Ophthalmol Vis Sci 2005; 46: 4182-4188.

3 Weinreb RN, Dreher AW, Coleman A, Quigley H, Shaw B, Reiter K. Histopathologic validation of Fourier-ellipsometry measurements of retinal nerve fiber layer thickness. Arch Ophthalmol 1990; 108: 557-560.

4 Zhou Q, Weinreb RN. Individualized compensation of anterior segment birefringence during scanning laser polarimetry. Invest Ophthalmol Vis Sci 2002; 43: 2221-2228.

5 Choplin NT, Zhou Q, Knighton RW. Effect of individualized compensation for anterior segment birefringence on retinal nerve fiber layer assessments as determined by scanning laser polarimetry. Ophthalmology 2003; 110: 719-725.

6 Greenfield DS, Knighton RW, Huang XR. Effect of corneal polarization axis on assessment of retinal nerve fiber layer thickness by scanning laser polarimetry. Am J Ophthalmol 2000; 129: 715-722.

7 Weinreb RN, Bowd C, Greenfield DS, Zangwill LM. Measurement of the magnitude and axis of corneal polarization with scanning laser polarimetry. Arch Ophthalmol 2002; 120: 901-906. 
8 Tannenbaum DP, Hoffman D, Lemij HG, Garway-Heath DF, Greenfield DS, Caprioli J. Variable corneal compensation improves discrimination between normal and glaucomatous eyes with the scanning laser polarimeter. Ophthalmology 2004; 111: 259-264.

9 Lleo-Perez A, Ortuno-Soto A, Rahhal MS, Martinez-Soriano F, Sanchis-Gimeno JA. Intraobserver reproducibility of retinal nerve fiber layer measurements using scanning laser polarimetry and optical coherence tomography in normal and ocular hypertensive subjects. Eur J Ophthalmol 2004; 14: 523-530.

10 Blumenthal EZ, Frenkel S. Inter-device reproducibility of the scanning laser polarimeter with variable cornea compensation. Eye 2005; 19: 308-311.
11 Frenkel S, Slonim E, Horani A, Molcho M, Barzel I, Blumenthal EZ. Operator learning effect and interoperator reproducibility of the scanning laser polarimeter with variable corneal compensation. Ophthalmology 2005; 112: 257-261.

12 Bagga H, Greenfield DS, Feuer WJ. Quantitative assessment of atypical birefringence images using scanning laser polarimetry with variable corneal compensation. Am J Ophthalmol 2005; 139: 437-446.

13 Da Pozzo S, Marchesan R, Canziani T, Vattovani O, Ravalico G. Atypical pattern of retardation on GDx-VCC and its effect on retinal nerve fibre layer evaluation in glaucomatous eyes. Eye 2006; 20: 769-775.

14 Altman DG, Royston P. The cost of dichotomising continuous variables. BMJ 2006; 332: 1080. 